\title{
In vitro modulation of probiotic bacteria on the biofilm of Candida glabrata
}

\begin{abstract}
A conspicuous new concept of pathogens living as the microbial societies in the human host rather than free planktonic cells has raised considerable concerns among scientists and clinicians. Fungal biofilms are communities of cells that possess distinct characteristic such as increased resistance to the immune defence and antimycotic agents in comparison to their planktonic cells counterpart. Therefore, inhibition of the biofilm may represent a new paradigm for antifungal development. In this study, we aim to evaluate the in vitro modulation of vulvovaginal candidiasis (VVC)-causing Candida glabrata biofilms using probiotic lactobacilli strains. Probiotic Lactobacillus rhamnosus GR-1 and Lactobacillus reuteri RC-14 were shown to have completely inhibited C. glabrata biofilms and the results were corroborated by scanning electron microscopy (SEM), which revealed scanty structures of the mixed biofilms of C. glabrata and probiotic lactobacilli strains. In addition, biofilmrelated C. glabrata genes EPA6 and YAK1 were downregulated in response to the probiotic lactobacilli challenges. The present study suggested that probiotic L. rhamnosus GR-1 and L. reuteri RC-14 strains inhibited C. glabrata biofilm by partially impeding the adherence of yeast cells and the effect might be contributed by the secretory compounds produced by these probiotic lactobacilli strains. Further investigations are required to examine and identify the biofilm inhibitory compounds and the mechanism of probiotic actions of these lactobacilli strains.
\end{abstract}

Keyword: Anti-biofilm; Candida glabrata; Lactobacillus rhamnosus GR-1; Lactobacillus reuteri RC-14; Probiotic; Vulvovaginal candidiasis 